\title{
Combining the Scattering Matrix and Spherical Harmonic Methods for Semiconductor Modeling
}

\author{
Zhiyi $\operatorname{Han}^{a}$, Neil Goldsman ${ }^{a}$ and Mark Stettler \\ ${ }^{a}$ Department of Electrical Engineering, University of Maryland, College Park, MD \\ 20742 \\ aEmail: zyhan@glue.umd.edu, neil@glue.und.edu Fax:+1-301-3149281 \\ Phone:+1-301-4053648 \\ ${ }^{6}$ Intel Corporation, Hillsboro, OR 97124
}

\begin{abstract}
The spherical harmonics (SH) are applied to realize the scattering matrix method of carrier transport modeling. Using the spherical harmonics has the advantage of reducing the matrix by approximately a factor of 10 less than the previous discrete basis approach[1]. To realize the approach, new sphericalharmonic functions are defined to overcome difficulties associated with orthogonality. The scattering matrix is calculated using Monte-Carlo(MC) techniques to populate the new basis functions. As a initial step towards demonstrating the accuracy of the new mathematical formulation, we calculate the electron energy distribution functions for different homogeneous fields. We also calculate the space-dependent average energy and velocity for a step electrical field, and show agreement with Monte Carlo simulations.
\end{abstract}

\section{Introduction}

In this work we show how to combine the Spherical Harmonic and Scattering Matrix approaches of semiconductor modeling in such a way as to take advantage of many of the attributes of both methods $[1,2,3,4,5]$.

\section{Theory and Realization}

In the 1-dimensional Scattering Matrix method, the device is divided into thin slabs. Modeling is then achieved by analyzing the effect the electric field and scattering have on the electrons in each slab. This is realized by partitioning the current into flows which are into and out of the slab from either side. Details of the current in momentum space in each slab are then obtained by further dividing the flow into numerous orthonormal components, and writing the current in terms of a flux vector. Transport through the slab can be described mathematically by operating on this flux vector with the Scattering Matrix. The flux vector can be expressed in terms of virtually any orthonormal basis set. The physical details of carrier flow in momentum space can then be explored by determining the flow associated with the individual 
constituent basis functions. The most direct method to resolve the flux is with a discrete momentum space basis $[1,2]$. Such a discretization can yield accurate results, but has the disadvantage of requiring considerable memory.

To overcome memory problems, we express the angular dependence of the flux vector in terms of continuous Spherical Harmonic basis functions. This is achieved by first expressing the flux function $F_{x}^{+}(k, \theta)$ as the product of two independent functions:

$$
F_{x}^{+}(k, 0)=\kappa(k) \Theta(0)
$$

For the variable $k$ we directly discretize on a uniform mesh, which assumes the orthogonal basis is composed of unit pulse functions $h_{i}(k)$, which are defined by

$$
h_{\imath}(k)= \begin{cases}1 & , k_{i} \leq k<k_{i+1} \\ 0 & , k<k_{i} \text { or } k_{i+1} \leq k\end{cases}
$$

The function $\kappa(k)$ is thus given by

$$
\kappa(k)=\sum_{i} \alpha_{i} h_{\imath}(k)
$$

where $\alpha_{i}$ is the coefficient of the unit pulse function $h_{i}(k)$.

For the angular dependence we use a revised Splıerical Harmonic-type expansion. This revision was necessary because otherwise orthogonality would force all SH coefficients with $l>0$ to be zero, and not allow higher order modes to be populated. We overcome this obstacle by incorporating the weight function into the basis function. The expansion for $\Theta(0)$ then becomes:

$$
\Theta(0)=\sum_{l=0,2, . .} \beta_{l} \tilde{P}_{l}(\theta) \quad 0 \leq 0<\frac{\pi}{2}
$$

Where we have defined the new Spherical Harmonic-type function $\tilde{P}_{l}(\theta)$ as

$$
\tilde{P}_{l}(\theta)=P_{l}(\theta) \sqrt{(2 l+1) \sin \theta}
$$

$\beta_{l}$ is an expansion coefficient, and $P_{l}(\theta)$ represents the standard Spherical Harmonics. The flux function, incident on the slab from the left $F_{x}^{+}(k, \theta)$, in terms of the revised Spherical Harmonics becomes

$$
F_{x}^{+}(k, 0)=\sum_{l} \sum_{i} C_{i l}^{+} h_{i}(k) \tilde{P}_{l}(0) \quad 0 \leq 0<\frac{\pi}{2}
$$

where $C_{i l}^{+}=\alpha_{i} \beta_{l}^{+}$. To fully describe the system, we will also have a flux $F_{x+d x}^{-}(k, 0)$ incident on the slab from the right, a flux $F_{x}^{-}(k, 0)$ transmitted to the left, and a flux $F_{x+d x}^{+}(k, 0)$ reflected to the right. These other 3 flux functions relative to the slab have analogous definitions. The goal of the analysis is to determine the expansion coefficients $C_{i l}$, and thus the flux function. To do so, we first must determine the scattering matrix.

The scattering matrix is determined by forcing all the incident particles to be in one particular mode, and then determining the modes of the particles which are transmitted and reflected from the slab. This is illustrated in Fig. 1. The scattering matrix is constructed by performing such a calculation for each incident mode. 


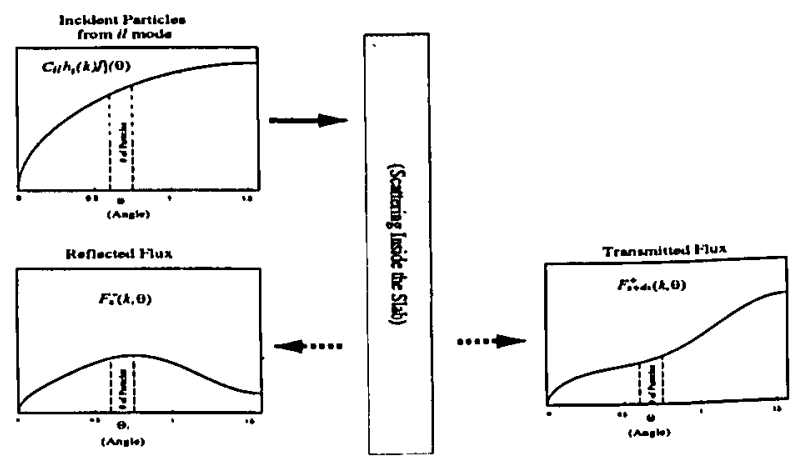

Figure 1: The Scattering of particles from a certain $i l$ mode and the transmitted and reflected particles fluxes of a specific momentum magnitude as a function of angle.

Once the scattering matrix is determined, the coefficients and thus the flux vectors are ascertained by solving the following equation iteratively.

$$
\left(\begin{array}{l}
\vec{C}_{n+1}^{+} \\
\vec{C}_{n+1}^{-}
\end{array}\right)=(\overline{\bar{M}})\left(\begin{array}{l}
\vec{C}_{n}^{+} \\
\vec{C}_{n}^{-}
\end{array}\right)
$$

After calculating the flux vectors, the distribution function can be readily obtained by dividing the flux by the momentum dependent velocity.

\section{Results}

As an initial demonstration of the approach, we used the method to solve the BTE for various homogeneous electric fields ranging from $1 \mathrm{kV} / \mathrm{cm}$ to $200 \mathrm{kV} / \mathrm{cm}$. We then compared the results to Monte Carlo calculations and found excellent agreement for all cases (Fig.2). We also compared the results of the new Spherical Harmonic basis with the previous descrete approach. We see in Fig. 3 that the two methods agree. However, the discrete approach requires a 10 times larger matrix. By cascading scattering matrices, we calculate the space-dependent average energy and velocity for a step field. In Fig.4 we show agrecment with MC for average energy and velocity, including the effects of overshoot.

\section{References}

[1] A.Das and M.S.Lundstrom,IEEE Trans. on Electron. Dev.,Vol.39,1992.

[2] M.A.Alam, M.A.Stettler and M.S.Lundstrom, Solid-State Electron. Vol.36, p.263, 1993.

[3] M.A.Alam, M.A.Stettler and M.S.Lundstrom,J. Appl. Phys. Vol.73, p.4998, 1993.

[4] Kenneth A.Hennacy, Y.-J. Wu, Neil Goldsman and Isaak Mayergoyz,Solid-State Electron.,Vol.38,p.1498,1995.

[5] Wenchao Liang, Neil Goldsman, Isaak Mayergoyz and Phil J. Oldiges, IEEE Trans. on Electron. Dev.,Vol.44, p.257-276,1997. 


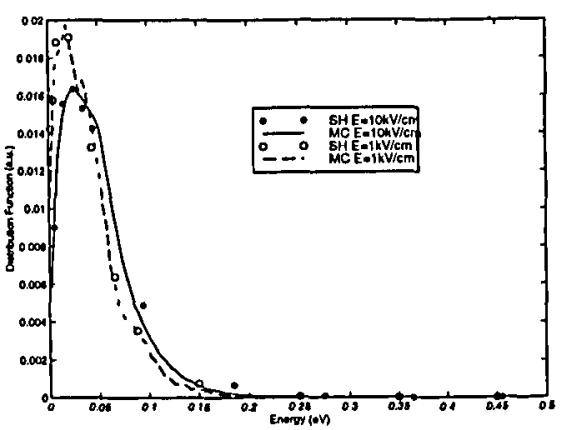

(a)

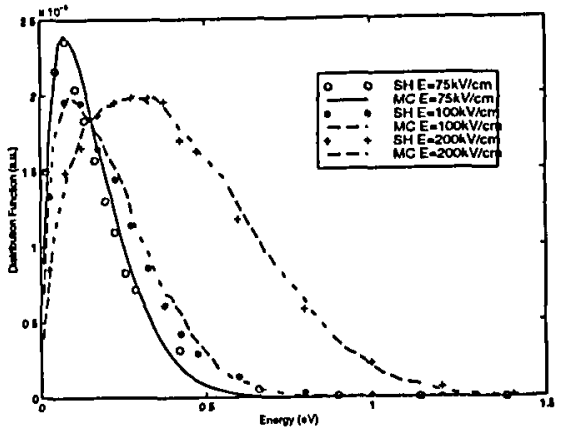

(b)

Figure 2: Distribution functions for electric fields of (a) $1 \mathrm{kV} / \mathrm{cm}, 10 \mathrm{kV} / \mathrm{cm}$ and (b) $75 \mathrm{kV} / \mathrm{cm}, 100 \mathrm{kV} / \mathrm{cm}, 200 \mathrm{kV} / \mathrm{cm}$ showing agreement between SH-SM and MC methods of calculation.

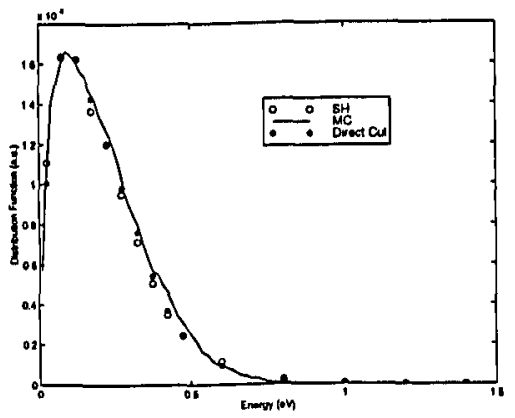

Figure 3: Distribution functions for electric field $100 \mathrm{kV} / \mathrm{cm}$ showing agreement between SH-SM and discrete basis Scattering Matrix approach.

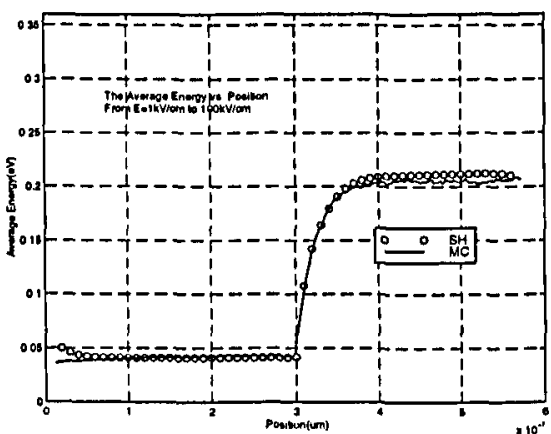

(a)

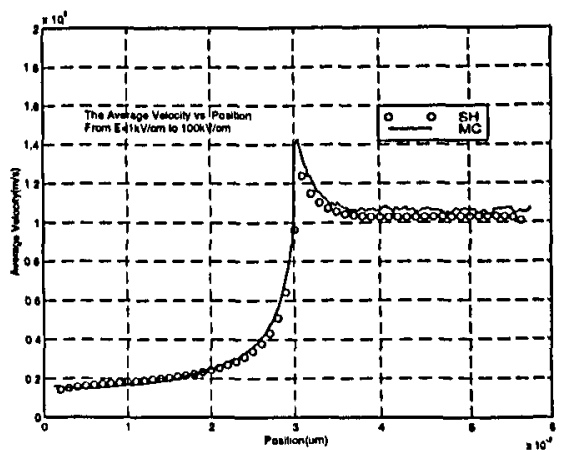

(b)

Figure 4: (a)The Average Energy vs Position as electrical field changes abruptly from $1 \mathrm{kV} / \mathrm{cm}$ to $100 \mathrm{kV} / \mathrm{cm}$ at $0.3 \mu \mathrm{m}$, (b) The Average Velocity vs Position as electrical field changes from $1 \mathrm{kV} / \mathrm{cm}$ to $100 \mathrm{kV} / \mathrm{cm}$. 